\begin{tabular}{|c|l|}
\hline Title & Sharp asymptotic behavior of solutions to nonlinear schrödinger equations with repul sive interactions \\
\hline Author(s) & Kita, Naoy asu; Ozawa, Tohru \\
\hline Citation & Hokkaido University Preprint Series in Mathematics, 612, 1-10 \\
\hline Issue Date & 2003-11-19 \\
\hline DOI & 10.14943/83757 \\
\hline Doc URL & http://hdl.handle.net/2115/69361 \\
\hline Type & bulletin (article) \\
\hline File Information & pre612.pdf \\
\hline
\end{tabular}

Instructions for use 


\title{
SHARP ASYMPTOTIC BEHAVIOR OF SOLUTIONS TO NONLINEAR SCHRÖDINGER EQUATIONS WITH REPULSIVE INTERACTIONS
}

\author{
NAOYASU KITA \\ Graduate School of Mathematics, Kyushu University, \\ Hakozaki 6-10-1, Higashi-ku, Fukuoka 812-8581, Japan \\ TOHRU OZAWA \\ Department of Mathematics, Hokkaido University, \\ Sapporo 060-0810, Japan
}

\begin{abstract}
A detailed description is given on the large time behavior of scattering solutions to the Cauchy problem for nonlinear Schrödinger equations with repulsive interactions in the short-range case without smallness condition on the data.
\end{abstract}

\section{Introduction}

We study the large time behavior of solutions to the Cauchy problem for the nonlinear Schrödinger equations of the form

$$
i \partial_{t} u+\frac{1}{2} \Delta u=f(u)
$$

where $u$ is a complex-valued function of $(t, x) \in \mathbf{R} \times \mathbf{R}^{\mathbf{n}}, \partial_{t}=\partial / \partial t, \Delta$ is the Laplacian in $\mathbf{R}^{\mathbf{n}}$, and $f$ denotes a nonlinear interaction given by a complex-valued function $f$ on C. In this paper we assume that $f$ is a single-power nonlinearity and satisfies the gauge invariance and repulsivity conditions and therefore we assume that $f$ takes the form

$$
f(u)=\lambda|u|^{p-1} u
$$

with $\lambda>0$ and $p>1$.

There is a large literature on the global Cauchy problem and the scattering theory for (1.1), see for instance $[2-18,20]$ and references therein. The usual scattering theory for (1.1) compares the full dynamics $\{u(t)\}$ given by solutions to (1.1) and the free dynamics 
described by the free propagator $U(t)=\exp (i(t / 2) \Delta)$. In the case of repulsive interactions the existence and asymptotic completeness of wave operators for (1.1) has been proved in the space $X_{1,1}=H^{1} \cap \mathcal{F}\left(H^{1}\right)$ for any $p$ with $\gamma(n) \leq p<\alpha(n)$, where $H^{1}$ is the Sobolev space of order one on $\mathbf{R}^{\mathbf{n}}$ defined by $H^{1}=(1-\Delta)^{-1 / 2} L^{2}\left(\mathbf{R}^{\mathbf{n}}\right), \mathcal{F}$ is the Fourier transform, $\gamma(n)=\left(n+2+\sqrt{n^{2}+12 n+4}\right) /(2 n)$, and $\alpha(n)=(n+2) /(n-2)$ if $n \geq 3$ and $\alpha(n)=\infty$ if $n \leq 2$. See $[10,20]$ for $p>\gamma(n)$ and $[2,3,15]$ for $p \geq \gamma(n)$. The exponent $\alpha(n)$ is usually referred to as the Sobolev critical exponent, while $\gamma(n)$ is sometimes referred to as the Strauss critical exponent $[4,16,17]$. The condition $p>\gamma(n)$ is equivalent to the integrability in time at infinity of the quantity $\left\|u(t) ; L^{p+1}\right\|^{p}$ of asymptotically free solutions $u(t)$ of $(1.1)$, namely $p \delta(p+1)>1$ with $\delta(r)=n / 2-n / r$. The last norm arises naturally in the energy for (1.1) and in the mapping property of $U(t): L^{r^{\prime}} \rightarrow L^{r}$ that has a conformity to the nonlinear mapping $u \mapsto|u|^{p-1} u$, where $t \neq 0, r \geq 2$, and $1 / r+1 / r^{\prime}=1$. Accordingly, equivalent conditions to $p \delta(p+1)>1$ appear in other equations such as wave and Klein-Gordon equations in various contexts $[16,17,18]$.

Methods of the proofs for the asymptotic completeness of wave operators depend on whether $p>\gamma(n)$ and $p=\gamma(n)$. The critical case $p=\gamma(n)$ requires special treatments. The method of [2,3] uses a contradiction argument with pseudo-conformal transformation which is unlikely to work for $n=2$, while the method of [15] is free from the pseudoconformal transformation and uses the Lorentz space in time where the borderline case $p \delta(p+1)=1$ is treated efficiently by means of homogeneity.

The purpose in this paper is to add further information on the large time asymptotics of scattering solutions for $p>\gamma(n)$. We present the second approximate term for scattering solutions. The term has first appeared in [13] (see also [14]) with several restrictive assumptions, such as smallness assumption on the data and restrictions on space dimensions as well as on the admissible range of powers in the nonlinearity. Here we remove those assumptions to some extent. Our proof uses Strichartz type inequalities and explicit integrability in time at infinity of scattering solutions. In this sense the method here has a close connection with the techinique for the case $p>\gamma(n)$.

To state our main theorem precisely, we introduce the following notation. For any $r$ with $1 \leq r \leq \infty, L^{r}=L^{r}\left(\mathbf{R}^{n}\right)$ denotes the Lebesgue space on $\mathbf{R}^{\mathbf{n}}$. For any $s \in \mathbf{R}$ and any $r$ with $1<r<\infty, H_{r}^{s}=(1-\Delta)^{-s / 2} L^{r}$ and $\dot{H}_{r}^{s}=(-\Delta)^{-s / 2} L^{r}$ denote the Sobolev space defined in terms of Bassel potentials and the homogeneous Sobolev space defined in terms of Riesz potentials, respectively. For any $s \in \mathbf{R}$ and any $r$ with $1 \leq r \leq \infty, \dot{B}_{r}^{s}=\dot{B}_{r, 2}^{s}$ denotes the homogeneous Besov space $[1,7,19]$. For any interval $I \subset \mathbf{R}$ and any Banach space $X$ we denote by $L^{g}(I ; X)$ or by $L_{t}^{g} X$ for simplicity the space of measurable functions $u$ form $I$ to $X$ such that $\|u(\cdot) ; X\| \in L^{g}(I)$. For $a, b \in \mathbf{R}$ we denote by $a \vee b$ and $a \wedge b$ 
the maximum and minimum, respectively. For the free propagator $U(t)=\exp (i(t / 2) \Delta)$ we use the factorization for $t \neq 0$

$$
U(t)=M(t) D(t) \mathcal{F} M(t)
$$

where $M(t)=\exp \left(i|x|^{2} /(2 t)\right)$ - is the modulation operator defined by the multiplication by $\exp \left(i|x|^{2} /(2 t)\right), D(t)$ is the dilation operator defined by $(D(t) \psi)(x)=(i t)^{-n / 2} \psi\left(t^{-1} x\right)$, and $\mathcal{F}$ is the Fourier transform defined by

$$
(\mathcal{F} \psi)(\xi)=\hat{\psi}(\xi)=(2 \pi)^{-n / 2} \int \exp (-i x \cdot \xi) \psi(x) \mathrm{d} x
$$

For any $\rho, \rho^{\prime} \in \mathbf{R}, X_{\rho, \rho^{\prime}}=H^{\rho^{\prime}} \cap \mathcal{F}\left(H^{\rho}\right)$ denotes the weighted Sobolev space and $\mathcal{X}_{\rho, \rho^{\prime}}$ denotes the associated function space for solutions to (1.1) defined by

$$
\begin{aligned}
\mathcal{X}_{\rho, \rho^{\prime}}=\left\{u \in C\left(\mathbf{R} ; X_{\rho, \rho^{\prime}}\right) ;\right. & u \in L_{\mathrm{loc}}^{q}\left(\mathbf{R} ; L^{r} \cap \dot{B}_{r}^{\rho^{\prime}}\right), M^{-1} u \in L_{\mathrm{loc}}^{q}\left(\mathbf{R} ; \dot{B}_{r}^{\rho}\right) \\
& \text { for any } q, r \text { with } 0 \leq 2 / q=\delta(r)<1\} .
\end{aligned}
$$

See [7] for basic facts and and related estimates on $\mathcal{X}_{\rho, \rho^{\prime}}$.

Theorem 1. Let $p$ and $n$ satisfy $\gamma(n)<p<\alpha(n)$ and $1 \leq n \leq 5$. Let $r_{0}=p+1$ and $\theta=n(p-1) / 2$. Let $q_{0}$ satisfy $2 / q_{0}=\delta\left(r_{0}\right)$.

(1) Suppose $n \leq 2$. Assume further that $p<3$ if $n=2$. Let $\phi \in X_{1,1}$. Let $u \in \mathcal{X}_{1,1}$ be the unique solution of (1.1) and (1.2) with $u(0)=\phi$. Let $\phi_{ \pm} \in X_{1,1}$ be the corresponding asymptotic states at $t= \pm \infty$, respectively. Then

$$
\begin{gathered}
\left\|u(t)-U(t) \phi_{ \pm}-V_{ \pm}(t) ; L^{2}\right\|=o\left(|t|^{1-\theta}\right) \\
\left\|u(t)-U(\cdot) \phi_{ \pm}-V_{ \pm} ; L^{q_{0}}\left(I_{T}^{ \pm} ; L^{r_{0}}\right)\right\|=o\left(T^{1-\theta}\right)
\end{gathered}
$$

as $t \rightarrow \pm \infty$ and $T \rightarrow+\infty$, where $I_{T}^{+}=[T, \infty), I_{T}^{-}=(-\infty,-T]$,

$$
V_{ \pm}(t)= \pm i(\theta-1)^{-1} t^{1-\theta} M(t) D(t) f\left(\hat{\phi}_{ \pm}\right)
$$

(2) Suppose $2 \leq n \leq 5$. Let $\rho$ satisfy $\delta\left(2 r_{0}\right) \vee \delta\left(p r_{0}\right) \vee\left(2 \delta\left(r_{0}\right)\right) \vee 1<\rho<p \wedge 2$. Let $\phi \in X_{\rho, 1}$. Let $u \in \mathcal{X}_{\rho, 1}$ be the unique solution of (1.1) and (1.2) with $u(0)=\phi$. Let $\phi_{ \pm} \in X_{\rho, 1}$ be the corresponding asymptotic states at $t= \pm \infty$, respectively. Then (1.3) and (1.4) hold.

Remark 1. (1) $\theta>1$ if and only if $p>1+2 / n$. Note that $1+2 / n<\gamma(n)<1+4 / n<$ $\alpha(n)$. 
(2) The condition $\delta\left(2 r_{0}\right)<p \wedge 2$ holds if $n \leq 5$. The condition $2 \delta\left(r_{0}\right)<p$ holds if $p<\alpha(n)$ and $n \leq 6$, while $2 \delta\left(r_{0}\right)<2$ if and only if $p<\alpha(n)$. The condition $\delta\left(p r_{0}\right)<p \wedge 2$ holds if $p<\alpha(n)$ and $n \leq 6$.

Remark 2. The existence of $u$ and $\phi_{ \pm}$in Part (1) has been proved in $[10,20]$. The existence of $u$ in Part (2) has been proved in [7]. The existence of $\phi_{ \pm}$in $X_{\rho, 1}$ shall be proved below.

Theorem 1 follows from the following two propositions.

Proposition 1. Let $p$ and $n$ satisfy $\gamma(n)<p<\alpha(n)$ and $n \geq 1$. Let $r_{0}, q_{0}$, and $\theta$ be as in Theorem 1. Let $\phi \in X_{1,1}$ and let $u \in \mathcal{X}_{1,1}$ be the unique solution of (1.1) and (1.2) with $u(0)=\phi$. Let $\phi_{ \pm} \in X_{1,1}$ be the corresponding asymptotic states at $t= \pm \infty$, respectively. Then (1.3) and (1.4) hold provided that

$$
\begin{aligned}
& \hat{\phi}_{ \pm} \in \dot{H}^{\delta(2 p)} \\
& f\left(\hat{\phi}_{ \pm}\right) \in L^{r_{0}} \cap \dot{H}_{r_{0}^{\prime}}^{2 \delta\left(r_{0}\right)+\varepsilon}
\end{aligned}
$$

with $\varepsilon>0$ sufficiently small.

Proposition 2. Let $p, n, r_{0}, q_{0}, \theta$ be as in Proposition 1. Let $\phi \in X_{1,1}$ and let $u \in \mathcal{X}_{1,1}$ be the unique solution of (1.1) and (1.2) with $u(0)=\phi$. Let $\phi_{ \pm} \in X_{1,1}$ be the corresponding asymptotic states at $t= \pm \infty$, respectively. Let $\rho$ satisfy $1 \leq \rho<p \wedge 2$. Assume further that $\phi \in X_{\rho, 1}$. Then $\phi_{ \pm} \in X_{\rho, 1}$ and $U(-t) u(t) \rightarrow \phi_{ \pm}$in $X_{\rho, 1}$ as $t \rightarrow \pm \infty$.

Remark 3. As for the restriction $\rho<2$, see[7].

We prove Proposition 1 in Section 2. The proof depends on an explicit representation of $u-U(\cdot) \phi_{ \pm}-V_{ \pm}$, the Strischartz estimates, and the $L^{r_{0}}$ decay estimate of scattering solutions. We prove Proposition 2 in Section 3. The method of proof depends on the argument in [7]. We prove Theorem 1 in Section 4. In the proofs below, we consider only the case $t>0$ since the case $t<0$ is treated similarly.

\section{Proof of Proposition 1.}

In this section we use the results in $[2,5,10,20]$ concerning scattering theory in $X_{1,1}$ for $p>\gamma(n)$. Let $u \in \mathcal{X}_{1,1}$ be the unique solution of (1.1) and (1.2) with $u(0)=\phi$. Then there exists a unique $\phi_{+} \in X_{1,1}$ such that $U(-t) u(t) \rightarrow \phi_{+}$in $X_{1,1}$ as $t \rightarrow \infty$. Moreover, $u$ satisfies the integral equation

$$
u(t)=U(t) \phi_{+}+i \int_{t}^{\infty} U(t-s) f(u(s)) \mathrm{d} s
$$


and the $L^{r_{0}}$ decay estimate

$$
\left\|u(t) ; L^{r_{0}}\right\| \leq C t^{-\delta\left(r_{0}\right)}
$$

We use (2.1) and the formula $U=M D \mathcal{F} M$ to represent $u-U(\cdot) \phi_{+}-V_{+}$as a sum of four integrals as

$$
\begin{aligned}
& u(t)-U(t) \phi_{+}-V_{+}(t) \\
& =i \int_{t}^{\infty} U(t-s) f(u(s)) \mathrm{d} s-i(\theta-1)^{-1} t^{1-\theta} U(t) M(-t) \mathcal{F}^{-1} f\left(\hat{\phi}_{+}\right) \\
& =i \int_{t}^{\infty} U(t-s)\left(f(u(s))-f\left(U(s) \phi_{+}\right)\right) \mathrm{d} s \\
& \quad+i \int_{t}^{\infty} U(t-s)\left(f\left(U(s) \phi_{+}\right)-s^{-\theta} M(s) D(s) f\left(\hat{\phi}_{+}\right)\right) \mathrm{d} s \\
& \quad+i U(t)\left(\int_{t}^{\infty} s^{-\theta} M(-s) \mathrm{d} s-(\theta-1)^{-1} t^{1-\theta} M(-t)\right) \mathcal{F}^{-1} f\left(\hat{\phi}_{+}\right) \\
& =i \int_{t}^{\infty} U(t-s)\left(\int_{0}^{1} f^{\prime}\left(\mu u(s)+(1-\mu) U(s) \phi_{+}\right) \mathrm{d} \mu\right)\left(u(s)-U(s) \phi_{+}-V_{+}(s)\right) \mathrm{d} s \\
& \quad+i \int_{t}^{\infty} U(t-s)\left(\int_{0}^{1} f^{\prime}\left(\mu u(s)+(1-\mu) U(s) \phi_{+}\right) \mathrm{d} \mu\right) V_{+}(s) \mathrm{d} s \\
& \quad+i \int_{t}^{\infty} U(t-s)\left(f\left(U(s) \phi_{+}\right)-f\left(M(s) D(s) \hat{\phi}_{+}\right)\right) \mathrm{d} s \\
& \quad+i M(t) D(t) \mathcal{F}^{2} \int_{t}^{\infty} s^{-\theta}(U(1 / s-1 / t)-1) \mathcal{F}^{-2} f\left(\hat{\phi}_{+}\right) \mathrm{d} s,
\end{aligned}
$$

where we have used the relations

$$
\begin{aligned}
& U(t) M(-t)\left(\int_{t}^{\infty} s^{-\theta} M(t) M(-s) \mathrm{d} s-(\theta-1)^{-1} t^{1-\theta}\right) \mathcal{F}^{-1} f\left(\hat{\phi}_{+}\right) \\
& =M(t) D(t)\left(\int_{t}^{\infty} s^{-\theta} \mathcal{F} M(t) M(-s) \mathcal{F}^{-1} f\left(\hat{\phi}_{+}\right) \mathrm{d} s-\int_{t}^{\infty} s^{-\theta} \mathrm{d} s f\left(\hat{\phi}_{+}\right)\right) \\
& =M(t) D(t) \mathcal{F}^{2} \int_{t}^{\infty} s^{-\theta}\left(\mathcal{F}^{-1} M(1 /(1 / t-1 / s)) \mathcal{F}-1\right) \mathcal{F}^{-2} f\left(\hat{\phi}_{+}\right) \mathrm{d} s \\
& =M(t) D(t) \mathcal{F}^{2} \int_{t}^{\infty} s^{-\theta}(U(1 / s-1 / t)-1) \mathcal{F}^{-2} f\left(\hat{\phi}_{+}\right) \mathrm{d} s .
\end{aligned}
$$

We denote by I, II, III, IV the first, second, third, fourth terms on the RHS of the last equality in (2.3). We estimate I, II, III in $L^{q_{0}}\left(T, \infty ; L^{r_{0}}\right)=L_{t}^{q_{0}} L^{r_{0}}$ by the Strichartz and Hölder inequalities. We first prove decay estimates similar to (2.2). Let $r$ satisfy $2 \leq r<\infty$. Then

$$
\begin{aligned}
\left\|U(t) \phi_{+} ; L^{r}\right\| & =\left\|D(t) \mathcal{F} M(t) \phi_{+} ; L^{r}\right\| \\
& =t^{-\delta(r)}\left\|\mathcal{F} M(t) \phi_{+} ; L^{r}\right\| \\
& \leq C t^{-\delta(r)}\left\|\mathcal{F} M(t) \phi_{+} ; \dot{H}^{\delta(r)}\right\| \\
& =C t^{-\delta(r)}\left\|\mathcal{F}|x|^{\delta(r)} M(t) \phi_{+} ; L^{2}\right\| \\
& =C t^{-\delta(r)}\left\||x|^{\delta(r)} \phi_{+} ; L^{2}\right\| \\
& =C t^{-\delta(r)}\left\|\hat{\phi}_{+} ; \dot{H}^{\delta(r)}\right\|,
\end{aligned}
$$

where we have used the Sobolev embedding $\dot{H}^{\delta(r)} \hookrightarrow L^{r}$. By the Strichartz and Hölder 
inequalities, we obtain

$$
\begin{aligned}
\left\|I ; L_{t}^{q_{0}} L^{r_{0}}\right\| & \leq C\left(\left\|u ; L_{t}^{q_{1}} L^{r_{0}}\right\|\left\|^{p-1}+\right\| U(\cdot) \phi_{+} ; L_{t}^{q_{1}} L^{r_{0}} \| p^{p-1}\right)\left\|u-U(\cdot) \phi_{+}-V_{+} ; L_{t}^{q_{0}} L^{r_{0}}\right\| \\
& \leq C T^{1-p \delta\left(r_{0}\right)}\left\|u-U(\cdot) \phi_{+}-V_{+} ; L_{t}^{q_{0}} L^{r_{0}}\right\|
\end{aligned}
$$

where $q_{1}=(p-1) /\left(1-\delta\left(r_{0}\right)\right)$ and we have used (2.2) and (2.4) with $r=r_{0}$.

Similarly, we have

$$
\begin{aligned}
\left\|\mathrm{II} ; L_{t}^{q_{0}} L^{r_{0}}\right\| & \leq C T^{1-p \delta\left(r_{0}\right)}\left\|V_{+} ; L_{t}^{q_{0}} L^{r_{0}}\right\| \\
& \leq C T^{1-p \delta\left(r_{0}\right)} \cdot T^{1-\theta-\delta\left(r_{0}\right) / 2}\left\|f\left(\hat{\phi}_{+}\right) ; L^{r_{0}}\right\| .
\end{aligned}
$$

Another use of the Strichartz inequalities implies

$$
\begin{aligned}
\left\|\mathrm{III} ; L_{t}^{q_{0}} L^{r_{0}}\right\| & \leq C\left\|f\left(U \phi_{+}\right)-f\left(M D \hat{\phi}_{+}\right) ; L_{t}^{1} L^{2}\right\| \\
& \leq C \int_{T}^{\infty}\left(\left\|U \phi_{+} ; L^{r}\right\|^{p-1}+\left\|M D \hat{\phi}_{+} ; L^{r}\right\|^{p-1}\right)\left\|U \phi_{+}-M D \hat{\phi}_{+} ; L^{m}\right\| \mathrm{d} t
\end{aligned}
$$

with $1 / 2=(p-1) / r+1 / m$. As in (2.4), we have

$$
\begin{aligned}
\left\|M D \hat{\phi}_{+} ; L^{r}\right\| & =t^{-\delta(r)}\left\|\hat{\phi}_{+} ; L^{r}\right\| \\
& \leq C t^{-\delta(r)}\left\|\hat{\phi}_{+} ; \dot{H}^{\delta(r)}\right\| \\
\left\|U \phi_{+}-M D \hat{\phi}_{+} ; L^{m}\right\| & =\left\|D \mathcal{F}(M-1) \phi_{+} ; L^{m}\right\| \\
& \leq C t^{-\delta(m)}\left\|(M-1)|x|^{\delta(m)} \phi_{+} ; L^{2}\right\| .
\end{aligned}
$$

We note here that $1 / 2=(p-1) / r+1 / m$ is equivalent to $(p-1) \delta(r)+\delta(m)=n(p-1) / 2=\theta$. Therefore, by (2.7), (2.8), and (2.9), we have

$$
\begin{aligned}
\left\|\mathrm{III} ; L_{t}^{q_{0}} L^{r_{0}}\right\| & \leq\left. C\left\|\hat{\phi}_{+} ; \dot{H}^{\delta(r)}\right\|\right|^{p-1} \int_{T}^{\infty} t^{-\theta}\left\|(M-1)|x|^{\delta(m)} \phi_{+} ; L^{2}\right\| \mathrm{d} t \\
& =o\left(T^{1-\theta}\right)
\end{aligned}
$$

as $T \rightarrow \infty$. We now choose $\delta(r)=\delta(m)$, which is equivalent to $r=m=2 p$. This in turn requires that $\hat{\phi}_{+} \in \dot{H}^{\delta(2 p)}$.

We consider IV, where $\mathcal{F}^{2}$ acts on functions as reflection. We take the $L_{t}^{q_{0}} L^{r_{0}}$ norm of IV. Since $q_{0} \delta\left(r_{0}\right)=2$, We have

$$
\begin{aligned}
& \left\|\mathrm{IV} ; L_{t}^{q_{0}} L^{r_{0}}\right\| \\
\leq & C\left(\int_{T}^{\infty} t^{-2}\left(\int_{t}^{\infty} s^{-\theta}\left\|(U(1 / s-1 / t)-1) \mathcal{F}^{-2} f\left(\hat{\phi}_{+}\right) ; L^{r_{0}}\right\| \mathrm{d} s\right)^{q_{0}} \mathrm{~d} t\right)^{1 / q_{0}}
\end{aligned}
$$

We now use the estimate

$$
\left\|(U(t)-1) \psi ; L^{r}\right\| \leq C m(t)^{\delta(r) / \delta\left(r_{1}\right)}\left\|\psi ; \dot{H}_{r^{\prime}}^{2 \delta(r) / \delta\left(r_{1}\right)}\right\|,
$$

where $m(t)=t^{1 / 2}$ for $n=1, m(t)=t^{1-\delta\left(r_{1}\right)}$ for $n \geq 2, \delta\left(r_{1}\right)=1 / 2$ for $n=1,0<\delta\left(r_{1}\right)<1$ for $n \geq 2$, and any $r$ with $0 \leq \delta(r) \leq \delta\left(r_{1}\right)$. The estimate (2.12) follows by interpolating 
between the following two estimates:

$$
\begin{aligned}
\left\|(U(t)-1) \psi ; L^{2}\right\| & \leq 2\left\|\psi ; L^{2}\right\|, \\
\left\|(U(t)-1) \psi ; L^{r_{1}}\right\| & \leq C \int_{0}^{t}\left\|U(s) \Delta \psi ; L^{r_{1}}\right\| \mathrm{d} s \\
& \leq C \int_{0}^{t} s^{-\delta\left(r_{1}\right)}\left\|\Delta \psi ; L^{r_{1}^{\prime}}\right\| \mathrm{d} s=C m(t)\left\|\psi ; \dot{H}_{r_{1}^{\prime}}^{2}\right\| .
\end{aligned}
$$

For $n \geq 2$, we take $\delta\left(r_{1}\right)$ sufficiently close to 1 and $r=r_{0}=p+1$. We put $\varepsilon=1-\delta\left(r_{1}\right)$. Then (2.12) yields

$$
\left\|(U(t)-1) \psi ; L^{r}\right\| \leq C t^{\varepsilon \delta\left(r_{0}\right) /(1-\varepsilon)}\left\|\psi ; \dot{H}_{r_{0}^{\prime}}^{2 \delta\left(r_{0}\right) /(1-\varepsilon)}\right\| .
$$

By (2.11) and (2.13), we continue the estimate on IV as

$$
\begin{aligned}
& \left\|\mathrm{IV} ; L_{t}^{q_{0}} L^{r_{0}}\right\| \\
& \leq C\left(\int_{T}^{\infty} t^{-2}\left(\int_{t}^{\infty} s^{-\theta}|1 / s-1 / t|^{\varepsilon \delta\left(r_{0}\right) /(1-\varepsilon)} \mathrm{d} s\right)^{q_{0}} \mathrm{~d} t\right)^{1 / q_{0}}\left\|\mathcal{F}^{-2} f\left(\hat{\phi}_{+}\right) ; \dot{H}_{r_{0}^{\prime}}^{2 \delta\left(r_{0}\right) /(1-\varepsilon)}\right\| \\
& \leq C T^{1-\theta-\delta\left(r_{0}\right) / 2-\varepsilon \delta\left(r_{0}\right) /(1-\varepsilon)}\left\|f\left(\hat{\phi}_{+}\right) ; \dot{H}_{r_{0}^{\prime}}^{2 \delta\left(r_{0}\right) /(1-\varepsilon)}\right\|,
\end{aligned}
$$

where we have used

$$
\begin{aligned}
& \int_{t}^{\infty} s^{-\theta}|1 / s-1 / t|^{\eta} \mathrm{d} s=\int_{0}^{1 / t} \sigma^{\theta-2}|1 / t-\sigma|^{\eta} \mathrm{d} \sigma \\
& =(1 / t)^{\theta-1+\delta} \int_{0}^{1} s^{\theta-2}(1-s)^{\eta} \mathrm{d} s=B(\theta-1, \eta+1) t^{1-\theta-\delta},
\end{aligned}
$$

where $\theta>1, \eta>-1$, and $B$ is the beta function. By (2.3), (2.5), (2.6), (2.10), and (2.14), we obtain

$$
\begin{aligned}
& \left\|u-U(\cdot) \phi_{+}-V_{+} ; L_{t}^{q_{0}} L^{r_{0}}\right\| \\
& \leq C T^{1-p \delta\left(r_{0}\right)}\left\|u-U(\cdot) \phi_{+}-V_{+} ; L_{t}^{q_{0}} L^{r_{0}}\right\|+o\left(T^{1-\theta}\right),
\end{aligned}
$$

from which we obtain

$$
\left\|u-U(\cdot) \phi_{+}-V_{+} ; L_{t}^{q_{0}} L^{r_{0}}\right\|=o\left(T^{1-\theta}\right) .
$$

By the Strichartz estimates, we may replace the norm on the RHS of (2.15) by any norm with admissible pair. In particular,

$$
\left\|u-U(\cdot) \phi_{+}-V_{+} ; L^{\infty}\left(T, \infty ; L^{2}\right)\right\|=o\left(T^{1-\theta}\right) .
$$

The proposition follows from (2.16) and (2.17).

\section{Proof of Proposition 2.}

We already know that for any $\phi \in X_{\rho, 1}$ the equation (1.1) with (1.2) has a unique global solution $u \in \mathcal{X}_{\rho, 1}$ with $u(0)=\phi$ (see [7]). It suffices to prove that $\phi_{+} \in X_{\rho, 0}$ and that 
$U(-t) u(t) \rightarrow \phi_{+}$in $X_{\rho, 0}$ as $t \rightarrow \infty$, since the asymptotic completeness holds in $X_{1,1}$ $[2,5,10,20]$.

Let $I=\left[T, T^{\prime}\right]$ with $0<T<T^{\prime}$. We estimate the function $I \ni t \mapsto|t|^{\rho} M(-t) u(t) \in$ $\dot{B}_{r_{0}}^{\rho}$ in $L^{q_{0}}(I)$ in the same way as in [7] with (2.2) as

$$
\begin{aligned}
& \left\||t|^{\rho} M^{-1} u ; L^{q_{0}}\left(I ; \dot{B}_{r_{0}}^{\rho}\right)\right\| \\
\leq & C\left\|\hat{\phi} ; \dot{H}^{\rho}\right\|+C\left\|u ; L^{q_{1}}\left(I ; L^{r_{0}}\right)\right\|^{p-1}\left\||t|^{\rho} M^{-1} u ; L^{q_{0}}\left(I ; \dot{B}_{r_{0}}^{\rho}\right)\right\| \\
\leq & C\left\|\hat{\phi} ; \dot{H}^{\rho}\right\|+C T^{1-p \delta\left(r_{0}\right)}\left\||t|^{\rho} M^{-1} u ; L^{q_{0}}\left(I ; \dot{B}_{r_{0}}^{\rho}\right)\right\|,
\end{aligned}
$$

where $q_{1}$ is as in (2.5) and we have used (2.2). By taking $T$ sufficiently large, we obtain

$$
\left\||t|^{\rho} M^{-1} u ; L^{q_{0}}\left(I ; \dot{B}_{r_{0}}^{\rho}\right)\right\| \leq C\left\|\hat{\phi} ; \dot{H}^{\rho}\right\|
$$

where $C$ is independent of $T^{\prime}$ as in (3.1). This implies that $|t|^{\rho} M^{-1} u \in L^{q_{0}}\left(T, \infty ; \dot{B}_{r_{0}}^{\rho}\right)$. Let $t>s>T$. Applying the Strichartz estimates again, we obtain

$$
\begin{aligned}
& \left\|U(-t) u(t)-U(-s) u(s) ; \mathcal{F}\left(\dot{H}^{\rho}\right)\right\| \\
= & \left\||J|^{\rho} \int_{s}^{t} U\left(t-t^{\prime}\right) f\left(u\left(t^{\prime}\right)\right) \mathrm{d} t^{\prime} ; L^{2}\right\| \\
= & \left\||t|^{\rho} M^{-1} \int_{s}^{t} U\left(t-t^{\prime}\right) f\left(u\left(t^{\prime}\right)\right) \mathrm{d} t^{\prime} ; \dot{H}^{\rho}\right\| \\
\leq & C\left\||t|^{\rho} M^{-1} \int_{s}^{t} U\left(t-t^{\prime}\right) f\left(u\left(t^{\prime}\right)\right) \mathrm{d} t^{\prime} ; L_{t}^{\infty}\left(s, \infty ; \dot{B}_{2}^{\rho}\right)\right\| \\
\leq & C\left\||t|^{\rho} M^{-1} f(u) ; L_{t}^{q_{0}^{\prime}}\left(s, \infty ; \dot{B}_{r_{0}^{\prime}}^{\rho}\right)\right\| \\
\leq & C\left\|u ; L_{t}^{q_{1}}\left(s, \infty ; L^{r_{0}}\right)\right\|\left\|^{p-1}\right\||t|^{\circ} M^{-1} u ; L_{t}^{q_{0}}\left(s, \infty ; \dot{B}_{r_{0}}^{\rho}\right) \| \\
\leq & C s^{1-p \delta\left(r_{0}\right)}\left\||t|^{\rho} M^{-1} u ; L_{t}^{q_{0}}\left(T, \infty ; \dot{B}_{r_{0}}^{\rho}\right)\right\| \\
\rightarrow & 0
\end{aligned}
$$

as $t>s \rightarrow \infty$. This implies that $\phi_{+} \in X_{\rho, 0}$ and that $U(-t) u(t) \rightarrow \phi_{+}$in $X_{\rho, 0}$ as $t \rightarrow \infty$. QED

\section{Proof of Theorem 1.}

The Case $n=1$. Let $\phi_{+} \in X_{1,1}$. Then in particular $\hat{\phi}_{+} \in H^{1}$. Then (1.5) follows by the Sobolev emdebedding $H^{1} \hookrightarrow L^{\infty}$ and by taking $\varepsilon>0$ to ensure that $2 \delta\left(r_{0}\right)+\varepsilon \leq 1$.

The Case $n=2, p<3$. In this case $2 \delta\left(r_{0}\right)<1$ if and only if $p<3$. We use the estimate

$$
\begin{aligned}
\left\|f\left(\hat{\phi}_{+}\right) ; H_{r_{0}^{\prime}}^{s}\right\| & \leq C\left\|f\left(\hat{\phi}_{+}\right) ; B_{r_{0}^{\prime}}^{s+\delta}\right\| \\
& \leq C\left\|\hat{\phi}_{+} ; L^{2 r_{0}}\right\|^{p-1}\left\|\hat{\phi}_{+} ; B_{2}^{s+\delta}\right\| \\
& \leq C\left\|\hat{\phi}_{+} ; \dot{H}^{\delta\left(2 r_{0}\right)}\right\|^{p-1}\left\|\hat{\phi}_{+} ; H^{s+\delta}\right\|
\end{aligned}
$$

with $0<s<s+\delta<2 \wedge p$ and $n \geq 1$, where we have used the usual embeddings between Besov and Triebel-Lizorkin spaces and nonlinear estimates in homogeneous Besov spaces [7]. We now take $\varepsilon, \delta>0$ to ensure that $2 \delta\left(r_{0}\right)+\varepsilon+\delta=s+\delta \leq 1$. 
The Case $2 \leq n \leq 5$. By Part (1) of Remark 1, we choose $\rho$ such that $\delta\left(2 r_{0}\right) \vee \delta\left(p r_{0}\right) \vee$ $\left(2 \delta\left(r_{0}\right)\right) \vee 1<\rho<p \wedge 2$. Then $\hat{\phi}_{+} \in \dot{H}^{\delta(2 p)}$ since $\delta(2 p)<\delta\left(2 r_{0}\right)$. Moreover, $f\left(\hat{\phi}_{+}\right) \in L^{r_{0}}$ since $\hat{\phi}_{+} \in \dot{H}^{\delta\left(p r_{0}\right)} \hookrightarrow L^{p r_{0}}$. In the same way as in (4.1), $f\left(\hat{\phi}_{+}\right) \in \dot{H}_{r_{0}^{\prime}}^{2 \delta\left(r_{0}\right)+\varepsilon}$ by taking $\varepsilon, \delta>0$ to ensure that $2 \delta\left(r_{0}\right)+\varepsilon+\delta=s+\delta \leq \rho$.

\section{References}

[1] J.Bergh, J Löfström, "Interpolation Spaces," Springer, Berlin / Heidelberg / New York, 1976.

[2] T. Cazenave, "Blow-up and Scattering in Nonlinear Schrödinger Equation," Textos de Métodos Matemáticos 30, Instituto de Matemática, Rio de Janeiro, 1994.

[3] T. Cazenave, F. B. Weissler, Rapidly decaying solutions of the nonlinear Schrödinger equation, Commun. Math. Phys. 147 (1992) 75-100.

[4] G. C. Dong, S. Li, On the initial value problem for a nonlinear Schrödinger equation, J. Differential Equations 42 (1981) 353-365.

[5] J. Ginibre, An introduction to nonlinear Schrödinger equations, in "Nonlinear Waves" (R. Agemi, Y. Giga, T. Ozawa, Eds.), GAKUTO International Series, Mathematical Sciences and Applications 10, Gakkōtosho, Tokyo 1997.

[6] J. Ginibre, "Introduction aux équations de Schrödinger non linéaires, Cours de DEA 1994-1995," Paris Onze édition L161, Orsay, 1998.

[7] J. Ginibre, T. Ozawa, G. Velo, On the existence of the wave operators for a class of nonlinear Schrödinger equations, Ann. Inst. Henri Poincaré, Physique Théorique 60 (1994) 211-239.

[8] J. Ginibre, G. Velo, On a class of nonlinear Schrödinger equations, I. The Cauchy problem, II. Scattering theory, J. Funct. Anal. 32 (1979) 1-71.

[9] J. Ginibre, G. Velo, Scattering theory in the energy space for a class of nonlinear Schrödinger equations, J. Math. Pures Appl. 64 (1985) 363-401.

[10] N. Hayashi, Y. Tsutsumi, Remarks on the scattering problem for nonlinear Schrödinger equations, Lecture Notes in Math., Springer, Berlin / Heidelberg / New York 1285 (1987) 162-168. 
[11] T. Kato, On nonlinear Schrödinger equations, Ann. Inst. Henri Poincaré, Physique Théorique 46 (1987) 113-129.

[12] T. Kato, Nonlinear Schrödinger equations, in "Schrödinger Operators" (H. Holden, A. Jensen, Eds.), Lecture Notes in Phys., Springer, Berlin / Heidelberg / New York 345 (1989) 218-263.

[13] N. Kita, Sharp $L^{r}$ asymptotics of the small solutions to the nonlinear Schrödinger equations, Nonlinear Anal. 52 (2003) 1365-1377.

[14] N. Kita, T. Wada, Sharp asymptotic behavior of solutions to nonlinear Schrödinger equations in one space dimension, Funkcialaj Ekvacioj 45 (2002) 53-69.

[15] K. Nakanishi, T. Ozawa, Remarks on scattering for nonlinear Schrödinger equations, NoDEA 9 (2002) 45-68.

[16] W. A. Strauss, Everywhere defined wave operators, in "Nonlinear Evolution Equations," Academic Press, New York (1978) 85-102.

[17] W. A. Strauss, Nonlinear scattering at low energy, J. Funct. Anal. 41 (1981) 110-133.

[18] W. A. Strauss, "Nonlinear Wave Equations," CBMS 73, AMS, Providence, 1989.

[19] H. Triebel, "Theory of Function Spaces," Birkhäuser, Basel, 1983.

[20] Y. Tsutsumi, Scattering problem for nonlinear Schrödinger equations, Ann. Inst. Henri Poincaré, Physique Théorique 43 (1985) 321-347.

[21] K. Yajima, Existence of solutions for Schrödinger evolution equations, Commun. Math. Phys. 110 (1987) 415-426. 